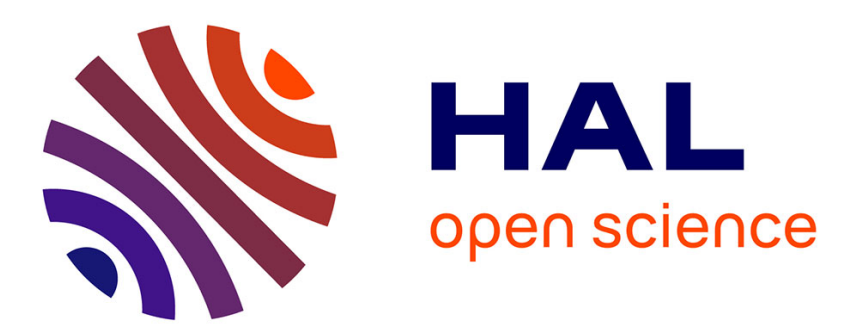

\title{
Evapotranspiration et caractéristiques aérodynamiques d'un gazon et d'une culture de riz en zone intertropicale humide
}

Jean-Paul Lhomme, Bruno Monteny, Joël Humbert

\section{- To cite this version:}

Jean-Paul Lhomme, Bruno Monteny, Joël Humbert. Evapotranspiration et caractéristiques aérodynamiques d'un gazon et d'une culture de riz en zone intertropicale humide. Agronomie, 1982, 2 (2), pp.183-186. hal-00884369

\section{HAL Id: hal-00884369 \\ https://hal.science/hal-00884369}

Submitted on 1 Jan 1982

HAL is a multi-disciplinary open access archive for the deposit and dissemination of scientific research documents, whether they are published or not. The documents may come from teaching and research institutions in France or abroad, or from public or private research centers.
L'archive ouverte pluridisciplinaire HAL, est destinée au dépôt et à la diffusion de documents scientifiques de niveau recherche, publiés ou non, émanant des établissements d'enseignement et de recherche français ou étrangers, des laboratoires publics ou privés. 


\title{
Evapotranspiration et caractéristiques aérodynamiques d'un gazon et d'une culture de riz en zone intertropicale humide
}

\author{
Jean-Paul LHOMME, Bruno MONTENY \& Joël HUMBERT \\ ORSTOM, Laboratoire de Bioclimatologie, B.P. V51, Abidjan, Côte-d'lvoire.
}

\section{RÉSUMÉ}

Evapotranspiration,

Gazon,

Riz pluvial,

Méthode mixte,

Paramètres aérodynami-

ques.

\begin{abstract}
La méthode mixte de détermination des flux est utilisée pour mesurer, sur la base de temps du quart d'heure, l'évapotranspiration réelle de deux couverts végétaux irrigués, l'un de gazon, l'autre de riz pluvial. Les caractéristiques aérodynamiques des deux couverts (rugosité, hauteur de déplacement, vitesse de frottement) sont analysées ainsi que l'évolution journalière de leur bilan énergétique. Les valeurs d'évaporation obtenues sont comparées aux valeurs correspondantes du terme radiatif de la formule de PENMAN (évapotranspiration standard, $\mathrm{ET}_{0}$ ). Dans les conditions dans lesquelles nous avons opéré, il apparaît que ces deux grandeurs sont relativement proches l'une de l'autre.
\end{abstract}

\section{SUMMARY}

Evapotranspiration,

Lawn,

Rice crop,

Combined method, Aerodynamic parameters.

\begin{abstract}
Evapotranspiration and aerodynamic parameters of a lawn and a rice crop in a intertropical humid region

The combined method (combination of the aerodynamic method and energy balance equation) is used to measure the evapotranspiration rate from two plant communities, a lawn and a rice crop. The aerodynamic parameters (roughness length, zero plane displacement, friction velocity) are analysed and their energy balance followed on a daily basis. We compare the measurements of evapotranspiration obtained by this method with the corresponding values of the radiative term of PENMAN's formula (standard evapotranspiration $\mathrm{ET}_{\mathrm{f}}$ ). Under our experimental conditions, the two estimates are relatively close in value.
\end{abstract}

\section{INTRODUCTION}

Cette étude présente et analyse des mesures de flux et d'évapotranspiration, réalisées près d'Abidjan $\left(5^{\circ} \mathrm{N}\right)$ en Côte-d'Ivoire. Ces mesures ont été effectuées sur deux couverts végétaux irrigués, l'un de gazon, l'autre de riz pluvial, par la méthode mixte, combinaison de la méthode aérodynamique et de celle du bilan d'énergie. En étudiant ces deux couverts, aux caractéristiques aérodynamiques et biologiques différentes, notre objectif a été d'obtenir une meilleure connaissance de l'ETR et de son estimation en climat intertropical humide.

Nous n'insisterons pas sur les aspects méthodologiques qui sont connus: la méthode aérodynamique mixte est maintenant un moyen classique de mesure de l'évapotranspiration, à la fois pratique et fiable (GRANT, 1975). Après avoir discuté les caractéristiques des deux couverts, et en particulier leurs propriétés aérodynamiques, et étudié l'évolution journalière de leur bilan énergétique, nous nous intéresserons à l'estimation de leur ETR au moyen de l'évapotranspiration standard (ou évapotranspiration d'équilibre) $E_{0}$. Beaucoup d'auteurs, en effet, à la suite de Slatyer \& MCIlroy (1961), Priestley \& Taylor (1972), ont montré l'intérêt de la prise en compte de ce paramètre dans l'estimation de l'ETR : citons, en France, les travaux de Bouchet \& PERrier (1973), qui ont discuté la signification de $\mathrm{ET}_{0}$ et en particulier son rôle limite, et ceux récents de Perrier et al. (1980) concernant une culture de blé dans le bassin parisien.

\section{MÉTHODE ET DISPOSITIF DE MESURE}

Le principe de la méthode aérodynamique mixte consiste à calculer le flux de chaleur sensible à partir des profils de vitesse du vent et de température et de déduire l'évaporation de l'équation du bilan d'énergie, grâce aux mesures du rayonnement net et du flux conductif de chaleur à la surface du sol. Son avantage sur les autres méthodes micrométéorologiques (rapport de BOWEN, méthode aérodynamique directe) vient du fait qu'elle ne nécessite pas de mesures d'humidité de l'air. Celles-ci sont en effet délicates à réaliser de façon continue dans les conditions naturelles (PERRIER $e t$ al., 1975).

\section{A. Conduite des calculs}

Les équations de base sont d'une part l'expression du bilan d'énergie (cf. tabl. 1) :

$$
\mathrm{Rn}+\Phi_{0}+\Phi_{\mathrm{s}}+\Phi_{\mathrm{L}}=0
$$


TABLEAU 1

Liste des symboles.

List of symbols.

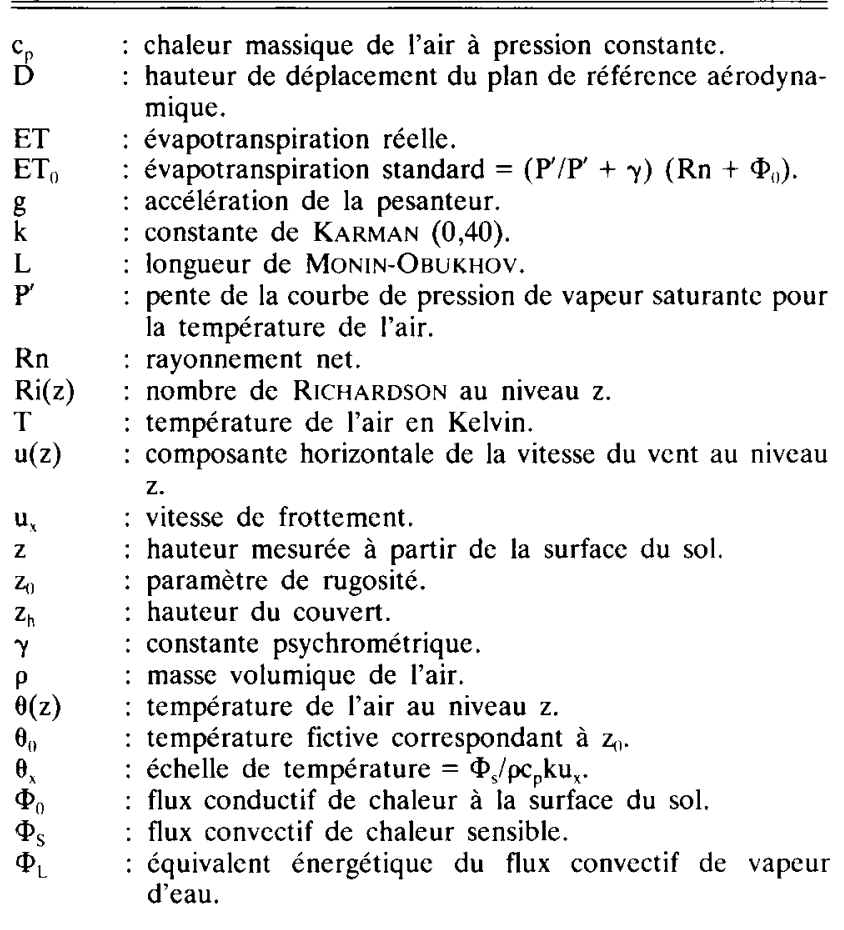

d'autre part, les expressions des profils de vitesse du vent et de température (PAulson, 1970; BuSINGER, 1975) :

$$
\begin{aligned}
& u(z)=\frac{u_{x}}{k}\left[\operatorname{Ln}\left(\frac{z-D}{z_{0}}\right)-F\left(\frac{z-D}{L}\right)\right] \\
& \theta(z)-\Theta_{0}=\frac{\Theta_{x}}{k}\left[\operatorname{Ln}\left(\frac{z-D}{z_{0}}\right)-G\left(\frac{z-D}{L}\right)\right]
\end{aligned}
$$

où $F$ et $G$ sont des fonctions de la variable adimensionnée $(z-D) / L$. L se calcule à partir du nombre de RICHARDSON au niveau $z$ en utilisant les relations suivantes:

$\mathrm{L}=(\mathrm{z}-\mathrm{D}) / \operatorname{Ri}(\mathrm{z})$

en régime d'instabilité thermique

$\mathrm{L}=(\mathrm{z}-\mathrm{D}) \cdot(1 / \operatorname{Ri}(\mathrm{z})-7)$

en régime de stabilité thermique.

Ri(z) est lui-même déterminé au moyen des gradients mesurés de vitesse du vent $(\Delta u)$ et de température $(\Delta \Theta)$ :

$\operatorname{Ri}(\mathrm{z})=\frac{\mathrm{g}}{\mathrm{T}} \cdot \frac{\partial \Theta / \partial \mathrm{z}}{(\partial \mathrm{u} / \partial \mathrm{z})^{2}}=\frac{\mathrm{g}}{\mathrm{T}} \cdot \frac{\Delta \Theta \cdot \Delta \mathrm{z}}{\Delta \mathrm{u}^{2}}$

De l'ajustement statistique, suivant la méthode des moindres carrés, des courbes théoriques (2) et (3) aux valeurs mesurées de la vitesse du vent et de la température, on déduit la valeur de la pente de chacune des droites de régression: $u(z)$ sur $[\operatorname{Ln}(z-D)-F(z-D)]$ et $\Theta(z)$ sur $[\operatorname{Ln}(z-D)-G(z-D)]$. Le flux de chaleur sensible s'obtient alors aisément à partir de $u_{x}$ et $\Theta_{x}: \Phi_{s}=\rho c_{p} u_{x} \Theta_{x}$. Comme D n'est pas connu à priori, on procède par tâtonnements pour le déterminer. Plusieurs valeurs de $\mathrm{D}$ sont essayées, d'abord pour calculer $\mathrm{L}$ à partir de $\mathrm{Ri}$, ensuite pour ajuster le profil théorique, et l'on retient celle qui donne le meilleur ajustement statistique, c'est-à- dire le meilleur coefficient de corrélation. Notons que ITIER (1980) et RIOU (1982) ont proposé des formules permettant d'estimer $\Phi_{\mathrm{s}}$ à partir de 2 niveaux de mesure seulement (la hauteur de déplacement $D$ étant fixée).

\section{B. Dispositif de mesure}

Les vitesses du vent sont mesurées à 6 hauteurs au-dessus du couvert avec des anémomètres à coupelles, type CASElLA. Le profil de température est déterminé grâce à une série de 5 thermocouples cuivre-constantan protégés du rayonnement direct par des petites plaques en bois. Un bilanmètre SCHENK mesure le rayonnement net et un fluxmètre (SRI 9), le flux conductif de chaleur.

Les tensions délivrées par tous ces capteurs sont intégrées sur une période de $12 \mathrm{mn}$ et les compteurs relevés pendant l'intervalle de $3 \mathrm{mn}$ qui termine le quart d'heure, base de temps servant à la mesure.

\section{CARACTERISTIQUES DES COUVERTS ÉTUDIÉS}

\section{A. Caractéristiques générales}

Deux couverts ont été étudiés sur le même site, près d'Abidjan, à des périodes voisines de l'année correspondant approximativement à la $2^{\mathrm{c}}$ moitié de la grande saison sèche. Chacun des 2 couverts avait une superficie de plus d'1 ha et était situé sur un terrain dégagé et approximativement horizontal. La région a un climat général de type intertropical humide avec un régime de vent de secteur S-SW (brise de mer). L'humidité de l'air est en permanence élevée $(\simeq 30 \mathrm{mb}$ ) même en saison sèche. Les mesures sur gazon ont été effectuées en mars et avril 1978 et, sur riz, en février et mars 1980. Les 2 couverts étaient régulièrement irrigués par aspersion.

Le gazon (Paspalum notatum) est un couvert pérenne avec fauches régulières. Sa hauteur évolue entre 8 à $20 \mathrm{~cm}$ environ entre chaque fauche. La culture de riz pluvial (variété "IRAT 13 », a été semée à la mi-décembre. Elle a atteint son indice foliaire maximal dès le stade de montaison (fin février-début mars). Durant la phase suivante, qui correspond au remplissage des grains, le gain de poids des panicules a été tel que l'épi s'est infléchi, réduisant ainsi la hauteur du couvert (de $1,05 \mathrm{~m}$, il est passé à $0,80 \mathrm{~m}$ ).

\section{B. Paramètres aérodynamiques}

Le tableau 2 donne les valeurs du paramètre de rugosité $\mathrm{z}_{0}$ en fonction de la hauteur du couvert. Dans les 2 cas, $\mathrm{z}_{0}$ est approximativement le même quelle que soit la hauteur : il est très voisin de $5 \mathrm{~cm}$ pour une culture de riz bien développée (après la montaison) et légèrement inférieur à $1 \mathrm{~cm}(0,8$ exactement) pour le gazon. Ces résultats recoupent assez bien ceux que l'on peut trouver dans la littérature scientifique. En ce qui concerne le riz, UCHIJIMA (1976) mentionne une dispersion assez importante dans la bande $3 \mathrm{~cm}-10 \mathrm{~cm}$. Pour le gazon, nos résultats sont en accord avec ceux de Gosse (1976) qui, pour le même couvert de gazon (Paspalum notatum), donne la relation :

$$
\mathrm{z}_{0}=8 \cdot 10^{-3} \pm 9 \cdot 10^{-4} \mathrm{~m} .
$$

Les 2 paramètres qui entrent directement dans le calcul du flux de chaleur sensible (et donc de l'évapotranspiration) sont la hauteur de déplacement du plan de référence 
TABLEAU 2

Paramètre de rugosité en fonction de la hauteur du couvert (moyenne et écart type par journée de mesure). Roughness length as a function of vegetation height (mean and standard deviation).

\begin{tabular}{|c|c|c|c|c|c|}
\hline \multicolumn{3}{|c|}{ Gazon } & \multicolumn{3}{|c|}{ Riz pluvial } \\
\hline Date (1978) & Hauteur $(\mathrm{cm})$ & $\bar{z}_{0} \pm \sigma(\mathrm{cm})$ & Date $(1980)$ & Hauteur $(\mathrm{cm})$ & $\overline{\mathrm{z}}_{0} \pm \sigma(\mathrm{cm})$ \\
\hline 15.03 & 20 & $0,91 \pm 0,30$ & 6.02 & 70 & $4,9 \pm 1,8$ \\
\hline 24.03 & 8 & $0,67 \pm 0,21$ & 25.02 & 85 & $5,1 \pm 1,9$ \\
\hline 30.03 & 11 & $0,90 \pm 0,10$ & 3.03 & 95 & $7,0 \pm 2,4$ \\
\hline 5.04 & 13 & $0,71 \pm 0,14$ & 10.03 & 100 & $4,8 \pm 1,8$ \\
\hline 6.04 & 15 & $0,70 \pm 0,25$ & 12.03 & 105 & $4,4 \pm 2,0$ \\
\hline 14.04 & 18 & $0,77 \pm 0,21$ & 17.03 & 85 & $4,8 \pm 2,4$ \\
\hline
\end{tabular}
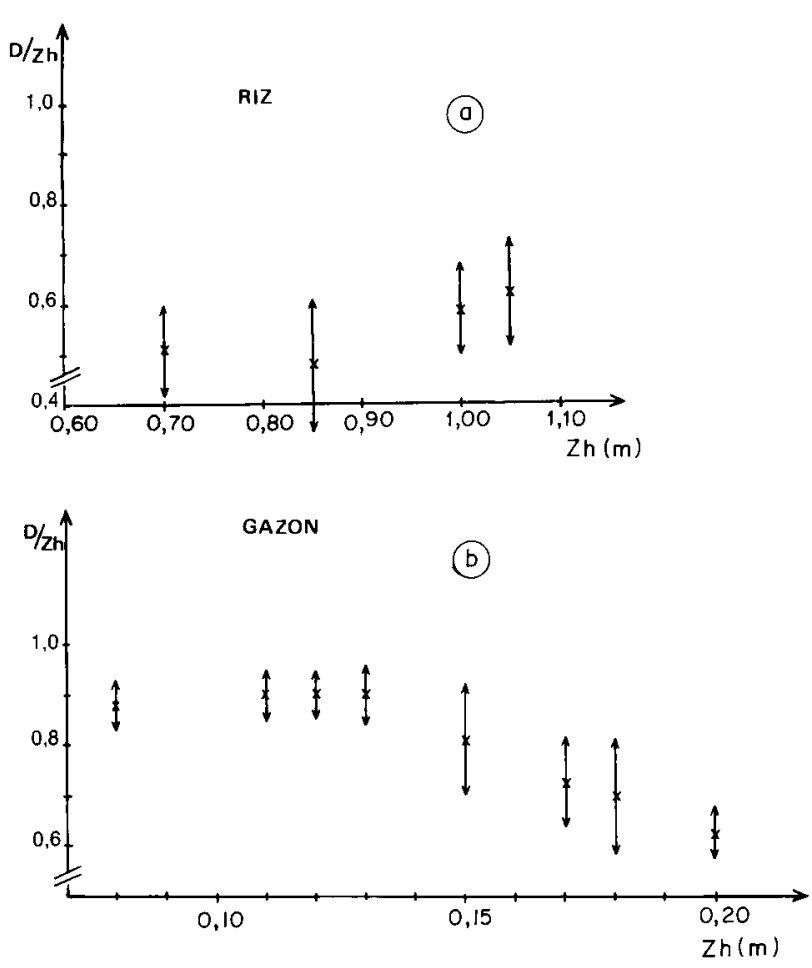

Figure 1

Evolution du rapport $D / z_{h}$ en fonction de la hauteur moyenne du couvert: $a:$ riz, $b:$ gazon.

Change in $D / z_{h}$ ratio with vegetation height : $a$ : rice crop, $b:$ lawn.

aérodynamique $(D)$ et la vitesse de frottement $\left(u_{\mathrm{x}}\right)$. D est lié à la hauteur $\mathrm{Zh}$ du couvert et caractérise sa plus ou moins grande perméabilité. La valeur $0,65-0,70$ du rapport $D / z_{h}$ est souvent utilisée (Тном, 1971). La figure 1 montre que, dans le cas du gazon, ce rapport diminue sensiblement avec la hauteur. Inversement, pour un couvert de riz bien développé, les résultats semblent indiquer une légère augmentation avec la hauteur (pas très nette, toutefois, si on considère la dispersion des valeurs). L'accroissement de la perméabilité dans le cas du gazon s'explique par le fait que les feuilles, en s'allongeant, s'assouplissent. Au contraire, les tiges de riz, en s'allongeant, acquièrent une certaine rigidité qui contribue, avec le développement simultané du feuillage, à une diminution de la perméabilité. Remarquons que les valeurs du rapport $\mathrm{D} / \mathrm{z}_{\mathrm{h}}$ sont dans le cas du gazon légèrement supérieures, et dans le cas du riz légèrement inférieures, à la valeur classiquement admise.

Nous n'avons pas mis en évidence de façon claire l'influence de la vitesse du vent sur D et $\mathrm{z}_{0}$. Dans le cas d'un couvert ras comme le gazon, l'impact de celle-ci sur ces 2 paramètres doit être somme toute faible. En ce qui concerne le riz, UCHIJIMA (1976) n'a pas trouvé, lui non plus, une dépendance nette vis-à-vis de la vitesse du vent.

Dans la figure 2 est précisée l'évolution de la vitesse de
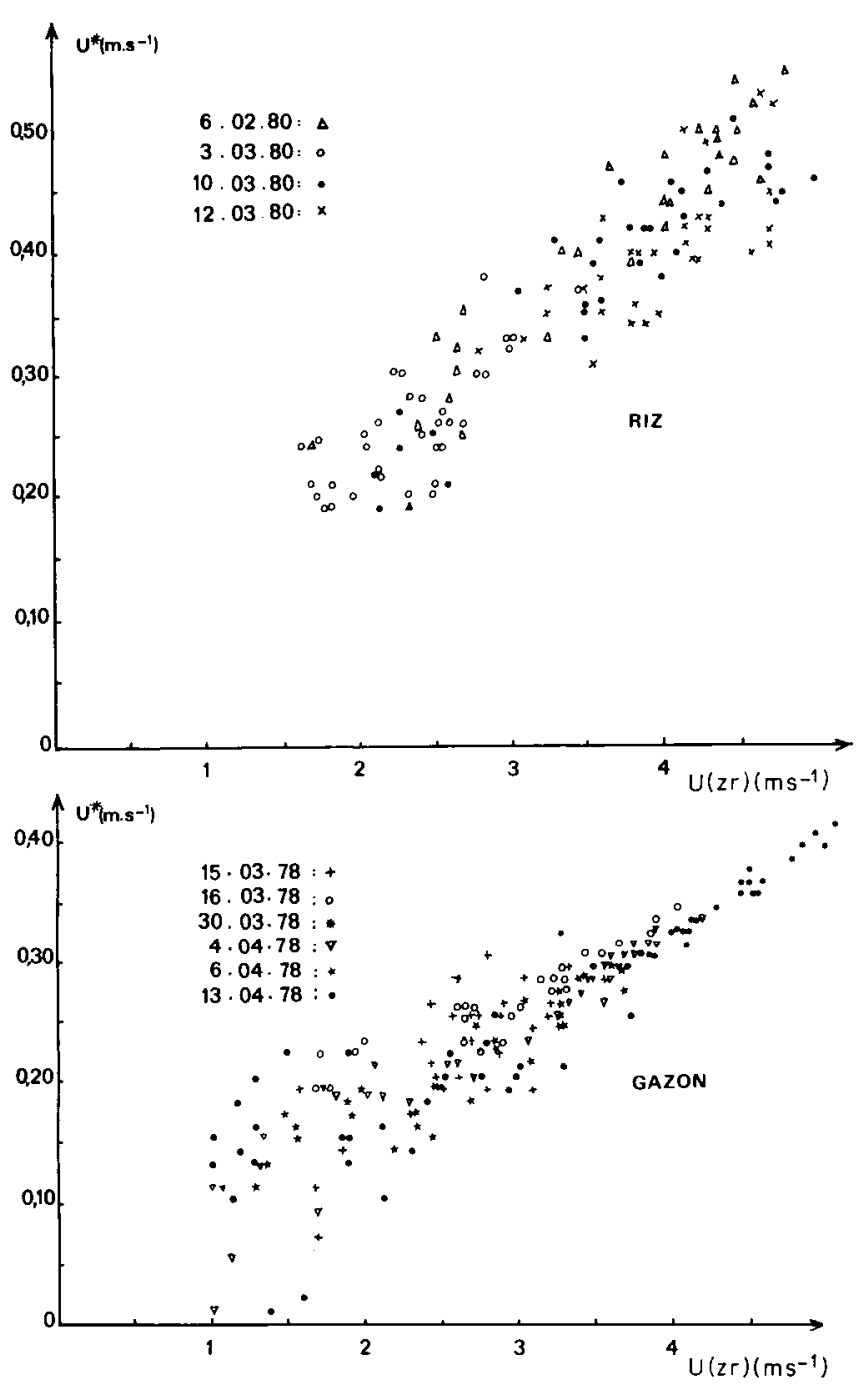

Figure 2

Evolution de la vitesse de frottement $u_{r}$ en fonction de la vitesse du vent au niveau de référence $\left(z_{r}\right)$ :

a) cas du riz $\left(z_{r}=3 \mathrm{~m}\right)$.

b) cas du gazon $\left(z_{r}=2,5 \mathrm{~m}\right)$.

Friction velocity $u_{x}$ as a function of wind speed at a reference level $\left(z_{r}\right)$ :

a) for the rice crop $\left(z_{r}=3 \mathrm{~m}\right)$.

b) for the lawn $\left(z_{r}=2,5 \mathrm{~m}\right)$. 
frottement $\left(u_{x}\right)$ en fonction de la vitesse du vent au niveau de référence $u\left(z_{r}\right)$. La relation est presque linéaire comme le laisse prévoir la théorie et la dispersion des points - qui est plus grande pour les faibles valeurs de vitesse du vent est en relation directe avec les phénomènes d'instabilité thermique, plus prononcés en régime de vent faible.

\section{IV. ÉVAPOTRANSPIRATION DES COUVERTS ÉTUDIÉS}

\section{A. Evolutions journalières de leur bilan énergétique}

La figure 3 présente 2 exemples d'évolution journalière des différents termes du bilan d'énergie sur Paspalum. La journée du 23 mars est caractérisée par un rayonnement net relativement faible $\left(<500 \mathrm{~W} \cdot \mathrm{m}^{2}\right)$. Le flux de chaleur sensible oscille autour de zéro (voisinage de la neutralité thermique) et l'évapotranspiration suit de très près le rayonnement net. L'énergie radiative disponible est donc presque entièrement consommée sous forme de chaleur latente de vaporisation. Durant la journée du 6 avril, le rayonnement net est plus élevé et l'instabilité thermique plus accentuée : l'évapotranspiration ne suit plus le rayonnement net.

La figure 4 montre, dans le cas du couvert de riz, l'évolution des différents flux au cours de 2 journées caractéristiques. Durant la journée du 3 mars $1980,6^{e}$ jour sans
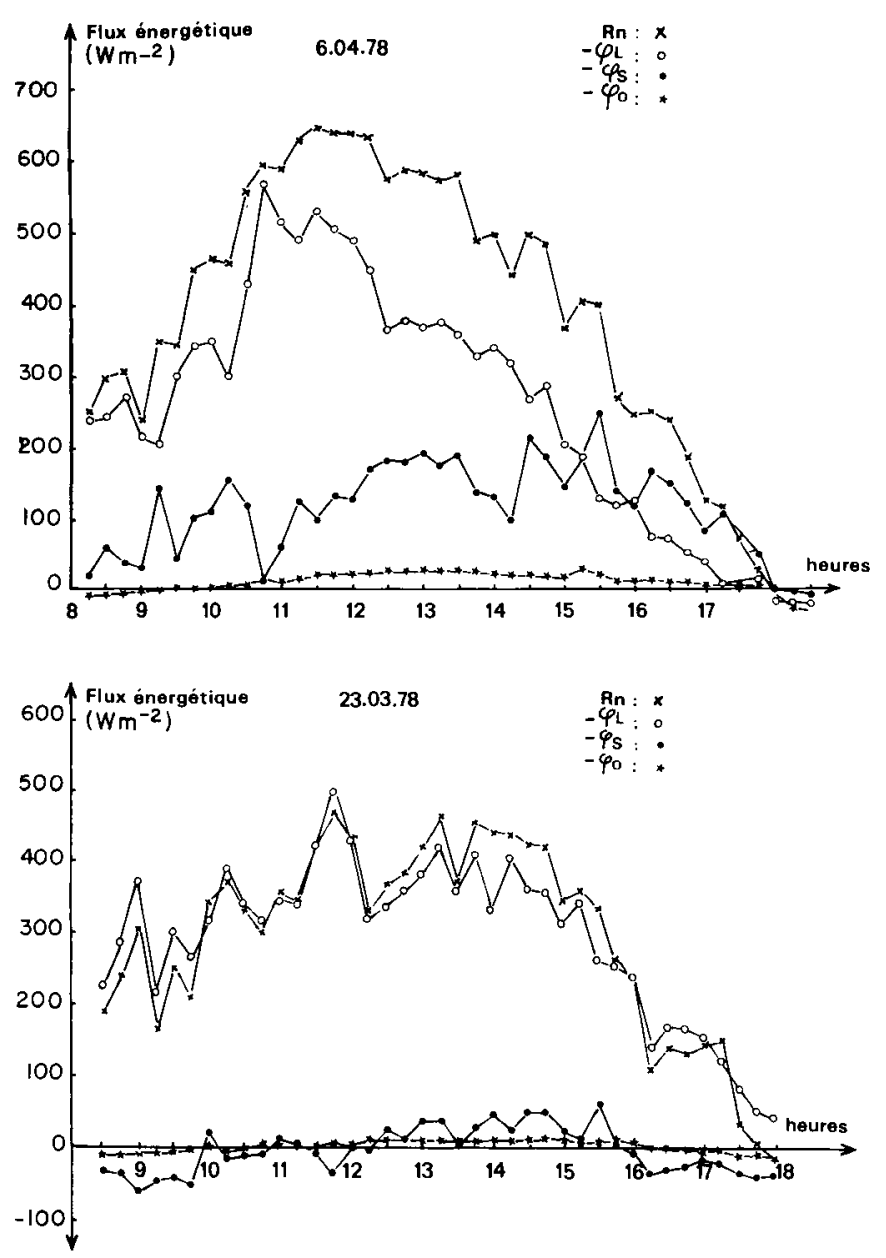

Figure 3

Exemples d'évolution journalière des différents termes du bilan d'énergie dans le cas du gazon.

Typical daily changes in the different components of the energy balance for lawn.
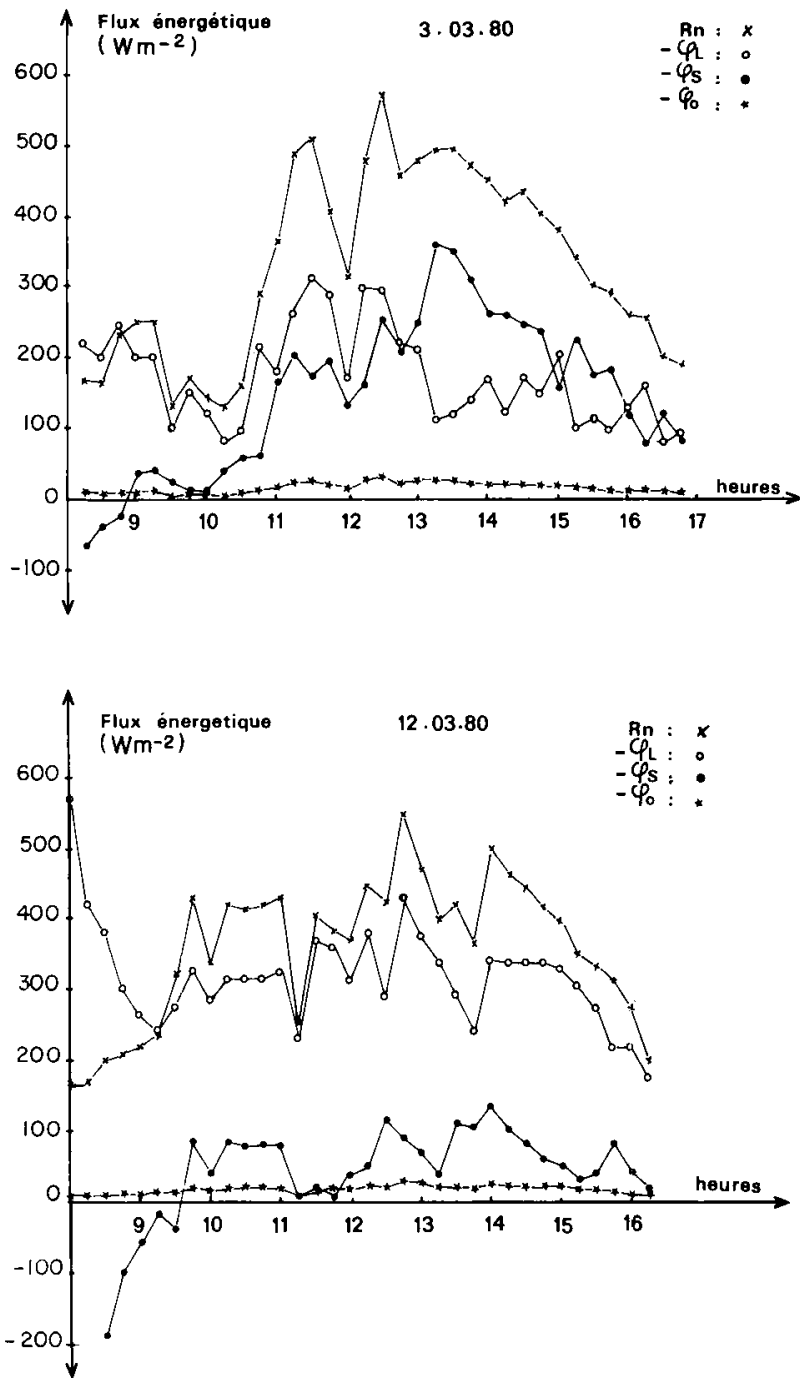

Figure 4

Exemples d'évolution journalière des différents termes du bilan d'énergie dans le cas du riz (avant irrigation le 3 mars et après irrigation le 12 mars).

Typical daily changes in the different components of the energy balance for the rice crop ( 3 march: before irrigation; 12 march : after irrigation).

pluie après une irrigation de $25 \mathrm{~mm}$, le couvert souffre d'un déficit hydrique assez prononcé, en particulier durant l'après-midi où $\left|\Phi_{\mathrm{S}}\right|>\left|\Phi_{\mathrm{L}}\right|$. Le 12 mars au lendemain d'une irrigation de $25 \mathrm{~mm}$, le flux de chaleur sensible est, au contraire, beaucoup plus faible et l'essentiel de l'énergie radiative est utilisé par l'évapotranspiration. Cette journée du 12 mars montre aussi un phénomène particulier en début de matinée, entre 8 h et 9 h 30 : l'évaporation intense de la rosée qui recouvre la culture par apport d'énergie advective. La masse d'air de secteur S-SW qui balaie à ce moment-là la culture, est relativement chaude (température voisine de $27^{\circ} \mathrm{C}$ ) et sa vitesse assez élevée (supérieure à $3 \mathrm{~m} \cdot \mathrm{s}^{-1}$ au niveau de référence) favorise le transfert convectif de chalcur. Ce phénomène s'observe également au cours d'autres journées, de façon un peu moins marquée.

\section{B. Expression climatique de l'évapotranspiration réelle}

Beaucoup d'auteurs à la suite de PRIESTLEY \& TAYloR (1972) s'accordent à penser que l'évapotranspiration des couverts végétaux bien alimentés en eau (régulation stomatique faible) dans des régions pas trop sèches s'éloigne peu 
de l'évapotranspiration d'équilibre $\mathrm{ET}_{0}$ (WILSON \& ROUSE, 1972) :

$\mathrm{ET}_{0}=\frac{\mathrm{P}^{\prime}}{\mathrm{P}^{\prime}+\gamma}\left(\mathrm{Rn}+\phi_{0}\right)$

L'évapotranspiration des couverts pourrait alors s'exprimer sous la forme $E T=k . E T_{0}$ où $k$ serait un coefficient voisin de 1 . Cette formulation relativement simple, qui réduit l'influence du climat à l'énergie radiative disponible (pondérée par la température), et résume celle de la culture dans un coefficient cultural $k$, a été largement utilisée depuis quelques années, dans de nombreuses conditions naturelles (DAVIES \& ALLEN, 1973 ; THOMPSON, 1975 ; GossE, 1976). Nous allons l'appliquer en Basse Côted'Ivoire, aux couverts considérés.
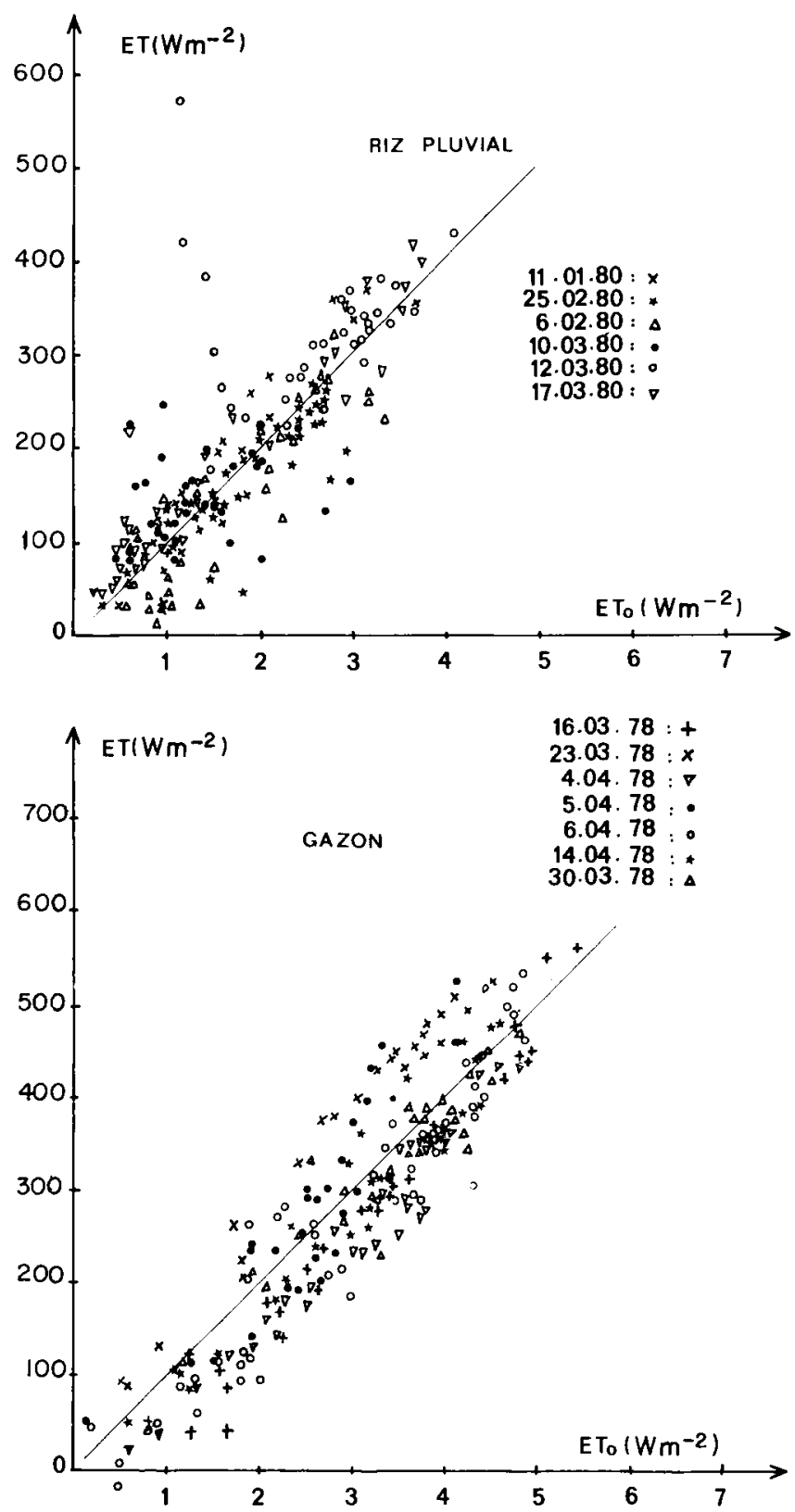

Figure 5

Evapotranspiration réelle (ET) en fonction de l'évapotranspiration standard $\left(E T_{0}\right)$ :

a) riz, b) gazon.

Actual evapotranspiration rate (ET) as a function of standard evapotranspiration $\left(E T_{0}\right)$ :

a) rice crop, b) lawn.
Voici dans le cas du gazon, les valeurs du coefficient $k$ obtenues pour quelques journées de mesure (moyenne des valeurs instantanées) :

$\begin{array}{lll}\text { - } 16 \text { mars 1978, } & \mathrm{z}_{\mathrm{h}}=0,20 \mathrm{~m}, & \mathrm{k}=0,86 \\ \text { - 23 mars 1978, } & \mathrm{z}_{\mathrm{h}}=0,08 \mathrm{~m}, & \mathrm{k}=1,26 \\ \text { - 30 mars 1978, } & \mathrm{z}_{\mathrm{h}}=0,11 \mathrm{~m}, & \mathrm{k}=0,96 \\ \text { - 04 avril 1978, } & \mathrm{z}_{\mathrm{h}}=0,13 \mathrm{~m}, & \mathrm{k}=0,82 \\ \text { - 05 avril 1978, } & \mathrm{z}_{\mathrm{h}}=0,13 \mathrm{~m}, & \mathrm{k}=1,08 \\ \text { - 06 avril 1978, } & \mathrm{z}_{\mathrm{h}}=0,14 \mathrm{~m}, & \mathrm{k}=0,91 \\ -14 \text { avril 1978, } & \mathrm{z}_{\mathrm{h}}=0,18 \mathrm{~m}, & \mathrm{k}=0,95\end{array}$

La valeur relativement élevée du coefficient $\mathrm{k}(1,26)$ le 23 mars, malgré un couvert peu développé s'explique par la proximité de l'irrigation (20 mars) et par une pluie de $6 \mathrm{~mm}$ tombée la veille. La valeur faible $(0,82)$ du 4 avril, qui succède à $\mathrm{k}=0,96$ le 30 mars, est due à l'assèchement progressif du sol après l'irrigation. Une pluie de $18 \mathrm{~mm}$ est tombée pendant la nuit du 4 au 5 avril. Le 5 , on retrouve un coefficient $\mathrm{k}$ de 1,08 . La figure 5 donne la répartition des points expérimentaux. Dans les conditions où nous avons opéré, l'évapotranspiration du gazon se situe bien au voisinage du terme $\mathrm{ET}_{0}$ autour duquel elle varie suivant les disponibilités en eau.

Dans le cas d'un couvert de riz bien développé et irrigué, nous observons également cette fluctuation autour de l'évapotranspiration standard :

$\begin{array}{lll}\text { - 06 février 1980, } & \mathrm{z}_{\mathrm{h}}=0,70 \mathrm{~m}, & \mathrm{k}=0,80 \\ \text { - } 25 \text { février } 1980, & \mathrm{z}_{\mathrm{h}}=0,85 \mathrm{~m}, & \mathrm{k}=0,95 \\ -03 \text { mars } 1980, & \mathrm{z}_{\mathrm{h}}=0,95 \mathrm{~m}, & \mathrm{k}=0,72 \\ -12 \text { mars } 1980, & \mathrm{z}_{\mathrm{h}}=1,05 \mathrm{~m}, & \mathrm{k}=1,22\end{array}$

La faible valeur du coefficient $\mathrm{k}$, le 3 mars, qui indique un certain stress hydrique, s'explique par le fait que le couvert n'avait pas reçu d'apport d'eau depuis $6 \mathrm{j}$ (dernière irrigation le 26 février). Au contraire, la forte valeur du 12 mars trouve son explication dans l'irrigation de la veille. La figure 5 montre la dispersion des résultats pour 6 journées de mesure. Les points correspondants au début de la journée du 12 mars (caractérisée par une évaporation importante de la rosée) se détachent nettement des autres points.

Les résultats que nous venons d'exposer, tout fragmentaires qu'ils soient, semblent montrer que l'évapotranspiration des couverts irrigués en Basse Côte-d'Ivoire s'éloigne peu du terme $\mathrm{ET}_{0}$. La variation de \pm 30 p. 100 observée est liée, en grande partie, à l'état de dessèchement du sol. Mais, dans leur ensemble, les valeurs que nous présentons sont inférieures à celles obtenues sur blé et maïs dans la région parisienne (KATERJ et al., 1977), où $\mathrm{k}$ avoisine 1,2 pour toute la période de végétation.

\section{CONCLUSION}

La méthode mixte, appliquée dans le cadre de cette étude, à deux couverts végétaux, l'un de gazon, l'autre de riz pluvial, nous a permis d'obtenir des valeurs d'évapotranspiration réelle dans le climat intertropical humide de la Basse Côte-d'Ivoire. Il apparaît que ces deux couverts irrigués, aux caractéristiques aérodynamiques différentes, ont globalement une évapotranspiration voisine de l'évapo- 
transpiration standard $\mathrm{ET}_{0}$. Ces résultats accréditent les idées avancées qui tendent à faire de $\mathrm{ET}_{0}$ la référence climatique en matière d'évapotranspiration (MONTENY et al., 1981). Elle est, en effet, très proche de l'ETR dans un grand nombre de conditions naturelles et fait intervenir des paramètres facilement calculables à partir des données météorologiques du réseau (MONTENY et al., 1980).

Reçu le 30 mars 1981. Accepté le 22 juin 1981.

\section{RÉFÉRENCES BIBLIOGRAPHIQUES}

Bouchet R. J., Perrier A., 1973. Communication au congrès « Le soleil au service de l'homme ». UNESCO. Paris. 29 p.

Businger J. A., 1975. Aerodynamics of vegetated surfaces. In "Heat and Mass transfer in the biosphere ». Part 1. D. de Vries and N. Afgan.

Davies J. A., Allen C. B., 1973. Equilibrium, potential and actual evaporation from cropped surfaces in Southern Ontario. J. appl. Meteorol., 12, 649-656.

Gosse G., 1976. Evaporation et caractéristiques d'échange d'un gazon en climat équatorial humide. Ann. agron., 27, 141-163.

Grant D. R., 1975. Comparison of evaporation measurements using different methods. Quart. J. r. meteorol. Soc., 101, 543-550.

Itier B., 1980. Une méthode simplifiée pour la mesure du flux de chaleur sensible. J. Rech. atmos., 14, 17-34.

Katerji N. B., Gosse G., Perrier A., Daudet F. A., 1977. Etude suivie de l'évapotranspiration réelle d'un couvert de blé et de maïs au moyen d'un dispositif automatique BEARN. La Météorologie, 6 (11), 47-53.

Monteny B., Lhomme J. P., 1980. Données climatiques recueillies à la Station OrSTOM d'Adiopodoumé, République de Côte-d'Ivoire. Rapport ORSTOM, $30 \mathrm{p}$.

Monteny B., Humbert J., Lhomme J. P., 1981. Le rayonnement net et l'estimation de l'évapotranspiration en Côte-d'Ivoire. Agric. Meteorol., 23, 45-59.

Paulson C. A., 1970. The mathematical representation of wind speed and temperature profiles in the instable atmospheric surface layer. J. appl. Meteorol., 9, 857-860.
Perrier A., Itier B., Bertolini J. M., Blanco de Pablos A., 1975. Mesure automatique du bilan d'énergie d'une culture, exemples d'application. Ann. agron., 26, 19-40.

Perrier A., Katerji N., Gosse G., Itier B., 1980. Etude in situ de l'évapotranspiration réelle d'une culture de blé. Agric. Meteorol., 21, 295-311.

Priestley C., Taylor P. A., 1972. On the assessment of surface heat and evaporation using large scale parameters. Month. Weather. Rev., 100, 81-92.

Riou C., 1982. Calcul du flux de chaleur sensible en conditions suradiabatiques à partir de mesures de vent et température à 2 niveaux. J. Rech. atmos., 16 (1). (sous presse).

Slatyer R. O., McIlroy I. C., 1961. Evaporation and the principle of its measurement. In «Practical Microclimatology». CSIRoUNESCO.

Thom A. S., 1971. Momentum absorption by vegetation. Quart. J. r. meteorol. Soc., 98, 124-134.

Thompson J. R., 1975. Energy budgets for three small plots substantiation of PRIESTLEY and TAYLOR's large-scale evaporation parameter. J. appl. Meteorol., 14, 1397-1401.

Uchijima Z., 1976́. Maize and rice. In «Vegetation and the atmosphere». Vol. 2. Monteith. Academic Press.

Wilson R. G., Rouse W. R., 1972. Moisture and temperature limits of the equilibrium evapotranspiration model. J. appl. Meteorol., 11, 436-442. 